\title{
Endothelin-1 induces oncostatin $M$ expression in osteoarthritis osteoblasts by trans-activating the oncostatin $M$ gene promoter via Ets-1
}

\author{
REN WU, WANCHUN WANG, GUOLIANG HUANG, XINZHAN MAO, YOU CHEN, QI TANG and LELE LIAO \\ Department of Orthopaedics, The Second Xiangya Hospital, Central South University, \\ Changsha, Hunan 410011, P.R. China
}

Received May 3, 2015; Accepted January 21, 2016

DOI: $10.3892 / \mathrm{mmr} .2016 .4960$

\begin{abstract}
Oncostatin M (OSM) contributes to cartilage degeneration in osteoarthritis (OA) and was demonstrated to be expressed in OA osteoblasts. Endothelin-1 (ET-1) is implicated in the degradation of OA articular cartilage, and osteoblast proliferation and bone development. In the present study, the effects of ET-1 on OSM expression in human OA osteoblasts were investigated, to the best of our knowledge, for the first time. Primary human OA osteoblasts were treated with ET-1 $(1,5,10,20$ and $30 \mathrm{nM})$ for $0.5,1,2,3$ and $4 \mathrm{~h}$ with or without the selective ETA receptor (ETAR) antagonist, BQ123, ETB receptor antagonist, BQ788 or the phosphatidylinositol 3-kinase (PI3K) inhibitor, BKM120. ET-1 treatment induced OSM mRNA expression, and the intracellular and secreted protein levels of OA osteoblasts in a dose-dependent manner. This effect was suppressed by BQ123 and BKM120, but not BQ788 administration. In combination with electrophoretic mobility shift assays, deletional and mutational analyses on the activity of a human OSM promoter/luciferase reporter demonstrated that ET-1 induced OSM expression in OA osteoblasts by trans-activating the OSM gene promoter through specific binding of Ets-1 to an Ets-1 binding site in the OSM promoter in an ETAR- and PI3K-dependent manner. Furthermore, ET-1 treatment increased the expression of Ets-1 in a dose-dependent manner, however the knockdown of Ets-1 suppressed the ET1-induced expression of OSM in OA osteoblasts. In conclusion, the present study demonstrated that ET-1 induces the expression of OSM in OA osteoblasts by trans-activating the OSM gene promoter primarily through increasing the expression level of Ets-1 in an ETAR- and
\end{abstract}

Correspondence to: Professor Wanchun Wang, Department of Orthopaedics, The Second Xiangya Hospital, Central South University, 139 Middle Renmin Road, Changsha, Hunan 410011, P.R. China

E-mail: wanchunwang61@yahoo.com

Key words: endothelin-1, oncostatin M, osteoarthritis, osteoblast, Ets-1, promoter, ETA receptor, phosphatidylinositol 3-kinase
PI3K-dependent manner. The current study suggested novel insights into the mechanistic role of ET-1 in the pathophysiology of $\mathrm{OA}$.

\section{Introduction}

Osteoarthritis (OA) is the most common age-associated degenerative joint disorder, and is a leading cause of pain and disability among older adults worldwide (1). A key process in $\mathrm{OA}$ is loss of the articular cartilage (1).

Oncostatin M (OSM) is a cytokine of the interleukin-6 (IL-6) family that is present at elevated levels in the synovial fluid of OA patients (2), and results in the secretion of pro-inflammatory cytokines, such as tumor necrosis factor- $\alpha$, IL-1 $\beta$ and IL-6, from osteoblasts and synovial cells that degrade the cartilage in arthritic joints (2). Previous studies demonstrated that OSM is associated with bone erosion, synovial inflammation and fibrosis, and cartilage degeneration (2-4).

Endothelin-1 (ET-1) is a potent vasoconstrictor that originates from vascular endothelial cells, and functions primarily through the activation of the ETA and ETB receptors (ETAR and ETBR, respectively) (5). Previous studies demonstrated that ET-1 has a role in the regulation of bone metabolism, stimulating the formation of new bone via osteoblastic proliferation (6,7). Previous studies demonstrated that ET-1 is involved in the degradation of osteoarthritic articular cartilage $(5,8)$, suggesting that ET-1 signaling may contribute to the destruction of the bone-cartilage unit in the pathophysiology of OA (5).

A previous study indicated that osteoblasts participate in the inflammation process in OA (9), and OSM was demonstrated to be expressed in osteoblasts isolated from the femurs of OA patients $(9,10)$. In the present study, the effect of ET-1 on the expression level of OSM in human OA osteoblasts was investigated, to the best of our knowledge, for the first time. Identification of this effect may contribute to elucidating the mechanistic role of ET-1 in the pathophysiology of OA.

\section{Materials and methods}

Reagents. Recombinant human ET-1, selective ETAR antagonist, BQ123, and selective ETBR antagonist, BQ788 were purchased 
from Sigma-Aldrich (St. Louis, MO, USA). The human OSM promoter/luciferase reporter and LightSwitch Luciferase Assay kit were purchased from SwitchGear Genomics (Shanghai, China). Mutant Osx promoter/luciferase reporter constructs were generated by polymerase chain reaction (PCR) and confirmed by sequencing. Life Technologies Lipofectamine 2000 transfection reagent, TRIzol reagent and SuperScript II reverse transcriptase were purchased from Thermo Fisher Scientific, Inc. (Waltham, MA, USA). Ets-1 (cat. no. sc-29309-V) and control (cat. no. sc-108080) shRNA lentiviral particles, selective phosphatidylinositol 3-kinase (PI3K) inhibitor, BKM120 (cat. no. sc-364437A), mouse monoclonal anti-OSM (E-4; cat. no. sc-365136), rabbit polyclonal anti-Ets1 (C-20; cat. no. sc-350) and mouse monoclonal anti-GAPDH (6C5; cat. no. sc-32233) antibodies were purchased from Santa Cruz Biotechnology, Inc. (Dallas, TX, USA). The OSM human ELISA kit (cat. no. ab100619) was purchased from Abcam (Shanghai, China). Putative transcription factor binding sites in the human OSM gene promoter sequence were identified using the online software, PROMO (http://alggen.lsi.upc.es) $(11,12)$.

Cell culture and treatment. Primary human OA osteoblasts and growth medium kit (cat. no. 406OAK-05a) were purchased from Cell Applications Inc. (San Diego, CA, USA). Cells were treated with $1,5,10,20$ or $30 \mathrm{nM}$ ET-1 for $0.5,1,2,3$ or $4 \mathrm{~h}$ in the presence or absence of BQ123 $(1 \mu \mathrm{M})$, BQ788 $(1 \mu \mathrm{M})$ or BKM120 $(10 \mu \mathrm{M})$. Cells were subjected to further analysis at $26-29.5 \mathrm{~h}$ after the treatment, making the total experimental time $30 \mathrm{~h}$. The medium remained unchanged.

Reverse transcription (RT)-quantitative (q) PCR. RNA was prepared from cells using the TRIzol reagent, and cDNAs were synthesized using SuperScript II reverse transcriptase. RT-qPCR was performed on an ABI PRISM 7700 Sequence Detection System, with fluorescent dye from the SYBR Green Master Mix (both Applied Biosystems; Thermo Fisher Scientific, Inc.) according to the manufacturer's instructions. PCR amplification condition were as follows: $20 \mathrm{sec}$ at $95^{\circ} \mathrm{C}$ followed by 40 cycles of $3 \mathrm{sec}$ at $95^{\circ} \mathrm{C}$ and $30 \mathrm{sec}$ at $60^{\circ} \mathrm{C}$. The primers used in the current study were as follows: Forward, 5'-AGAGTACCGCGTGCTCCTT-3' and reverse, 5'-AGC TTGCGCTGAAAAGCAT-3' for OSM; forward, 5'-GGG TGACGACTTCTTGTTTG-3' and reverse, 5'-GTTAAT GGAGTCAACCCAGC-3' for Ets-1; forward, 5'-GACTCA TGACCACAGTCCATGC-3' and reverse, 5'-AGAGGCAGG GATGATGTTCTG-3' for GAPDH. Relative quantification of the mRNA level was determined using the $2^{\Delta \Delta C q}$ method (13), which normalizes the expression levels of OSM or Ets-1 against that of GAPDH in the same samples. The mRNA level of treated cells was demonstrated as the fold changes to that of untreated control cells (designated as 1). Each experiment was repeated three times in duplicate.

ELISA and western blot analyses. The secreted OSM levels in cell culture supernatants were determined using an ELISA kit according to the manufacturer's instructions, normalized against cell number (per $10^{5}$ cells), and demonstrated as the fold changes to that of the untreated control cells (designated as 1). Each ELISA experiment was repeated three times in duplicate. For western blot analysis, human osteoblasts were lysed by three rounds of 3 -sec sonication on ice, with a hypotonic buffer, containing $2 \%$ Nonidet-P and a protease inhibitor cocktail (Sigma-Aldrich). The supernatant obtained following centrifugation at $2,000 \mathrm{xg}$ for $15 \mathrm{~min}$ at $4^{\circ} \mathrm{C}$ was used to determine the protein concentration with the Coomassie Blue staining (Thermo Fisher Scientific, Inc.). Equal quantities of proteins were separated by $10 \%$ SDS-polyacrylamide gel (Thermo Fisher Scientific, Inc.) at $100 \mathrm{~V}$ for $2 \mathrm{~h}$ and blotted onto a polyvinylidene difluoride microporous membrane (EMD Millipore, Billerica, MA, USA). Membranes were incubated for $1 \mathrm{~h}$ with a 1:1,000 dilution of the primary antibodies, washed thrice for $5 \mathrm{~min}$ and incubated for $1 \mathrm{~h}$ at room temperature with 1:5,000 dilution of secondary antibodies, including bovine anti-rabbit immunoglobulin $\mathrm{G}$ (IgG)-horseradish peroxidase (HRP; cat. no. sc-2370) or bovine anti-mouse IgG-HRP (cat. no. sc-2371; both Santa Cruz Biotechnology, Inc.). Peroxidase activity was revealed using an ECL kit (GE Healthcare Life Sciences, Shanghai, China). Three independent experiments were performed.

Transient transfection and luciferase assay. Cells were transfected with human OSM promoter/luciferase reporter plasmids using Lipofectamine 2000 transfection reagent. The luciferase assays were performed $30 \mathrm{~h}$ after transfection using the LightSwitch Luciferase Assay kit according to the manufacturer's instructions. The pRL-CMV plasmid (Promega Corporation, Madison, WI, USA) encoding Renilla reniformis luciferase (at one-fifth molar ratio to test plasmids) was co-transfected with test plasmids in each transfection as an internal control for data normalization. Each experiment was repeated three times in duplicate.

Electrophoretic mobility shift assay (EMSA). Nuclear extracts were prepared as previously described (14). EMSA was performed with 32P-labeled double-stranded oligonucleotides that were incubated with nuclear extract in EMSA buffer [10 mM Tris (pH 7.5), $5 \%$ glycerol, $1 \mathrm{mM}$ EDTA (pH 7.1), $50 \mathrm{mM} \mathrm{NaCl}, 1 \mathrm{mM}$ DTT, $1 \mathrm{mM}$ EDTA and $0.1 \mathrm{mg} / \mathrm{ml}$ poly (dI-dC); Sigma-Aldrich]. For the oligonucleotide competition analysis, a 100-fold molar excess of unlabeled competitor oligonucleotide was added to the mixture and incubated at room temperature for $30 \mathrm{~min}$. For the antibody supershift assays, monoclonal Ets-1 antibody $(1 \mu \mathrm{l})$ was added to the mixture. The reaction was then incubated on ice for $1 \mathrm{~h}$. Protein-DNA complexes and free DNA were fractionated on 5\% polyacrylamide gels in 1X Tris-glycine EDTA buffer (both Sigma-Aldrich) at $4^{\circ} \mathrm{C}$ and were visualized with a Cyclone Plus Phosphor Imager (C431200; Perkin Elmer, Inc., Waltham, MA, USA).

Lentiviral transduction. The Ets-1 shRNA lentiviral particles contained expression constructs encoding target-specific 19-25 nt (plus hairpin) shRNA designed to specifically knockdown the Ets-1 gene expression. The control shRNA lentiviral particles contained a scrambled shRNA sequence that would not lead to degradation of any cellular mRNA, and served as a negative control for the Ets-1 shRNA lentiviral particles. Lentiviral transduction was performed in the cells 
Table I. Oncostatin M mRNA levels in human OA osteoblasts treated with ET-1 and ET receptor antagonists.

Time (h)

\begin{tabular}{llllll} 
ET-1 $(\mathrm{nM})$ & \multicolumn{1}{c}{0.5} & \multicolumn{1}{c}{1} & \multicolumn{1}{c}{2} & 3 & 4 \\
\hline 1 & $1.02 \pm 0.03$ & $1.03 \pm 0.04$ & $1.05 \pm 0.05$ & $1.07 \pm 0.05$ & $1.08 \pm 0.07$ \\
5 & $1.07 \pm 0.05$ & $1.20 \pm 0.07^{\mathrm{a}, \mathrm{d}}$ & $1.59 \pm 0.1^{\mathrm{a}, \mathrm{d}, \mathrm{e}}$ & $2.13 \pm 0.14^{\mathrm{a}, \mathrm{d}-\mathrm{f}}$ & $2.42 \pm 0.17^{\mathrm{a}, \mathrm{d}-\mathrm{f}}$ \\
10 & $1.15 \pm 0.06^{\mathrm{a}}$ & $1.59 \pm 0.11^{\mathrm{a}, \mathrm{b}, \mathrm{d}}$ & $2.36 \pm 0.15^{\mathrm{a}, \mathrm{b}, \mathrm{d}, \mathrm{e}}$ & $2.94 \pm 0.19^{\mathrm{a}, \mathrm{b}, \mathrm{d}-\mathrm{f}}$ & $3.33 \pm 0.21^{\mathrm{a}, \mathrm{b}, \mathrm{d}-\mathrm{f}}$ \\
20 & $1.34 \pm 0.08^{\mathrm{a}, \mathrm{b}}$ & $2.41 \pm 0.15^{\mathrm{a}-\mathrm{d}}$ & $3.20 \pm 0.20^{\mathrm{a}-\mathrm{e}}$ & $3.71 \pm 0.23^{\mathrm{a}-\mathrm{f}}$ & $3.88 \pm 0.21^{\mathrm{a}-\mathrm{f}}$ \\
30 & $1.51 \pm 0.12^{\mathrm{a}-\mathrm{c}}$ & $2.67 \pm 0.18^{\mathrm{a}-\mathrm{d}}$ & $3.26 \pm 0.21^{\mathrm{a}-\mathrm{e}}$ & $3.78 \pm 0.22^{\mathrm{a}-\mathrm{f}}$ & $3.90 \pm 0.20^{\mathrm{a}-\mathrm{f}}$ \\
$30+$ BQ123 $(1 \mu \mathrm{M})$ & $0.95 \pm 0.05$ & $0.97 \pm 0.06$ & $1.02 \pm 0.09$ & $1.04 \pm 0.1$ & $1.05 \pm 0.12$ \\
$30+$ BQ788 $(1 \mu \mathrm{M})$ & $1.45 \pm 0.12$ & $2.56 \pm 0.19^{\mathrm{a}-\mathrm{d}}$ & $3.21 \pm 0.23^{\mathrm{a}-\mathrm{e}}$ & $3.75 \pm 0.25^{\mathrm{a}-\mathrm{f}}$ & $3.89 \pm 0.28^{\mathrm{a}-\mathrm{f}}$ \\
$30+$ BKM120 $(10 \mu \mathrm{M})$ & $1.06 \pm 0.1$ & $1.09 \pm 0.12$ & $1.14 \pm 0.13$ & $1.17 \pm 0.15$ & $1.19 \pm 0.16$ \\
\hline
\end{tabular}

OSM mRNA level in ET-1-treated human OA osteoblasts demonstrated as fold changes to that of the untreated control cells (designated as 1). P<0.05 vs. : ${ }^{\mathrm{E}} \mathrm{ET}-1(1 \mathrm{nM}) ;{ }^{\mathrm{b}} \mathrm{ET}-1(5 \mathrm{nM}) ;{ }^{\mathrm{C}} \mathrm{ET}-1$ (10 nM); ${ }^{\mathrm{d}} 0.5$-h ET-1 treatment $(1,5,10,20$ or $30 \mathrm{nM})$; ${ }^{\mathrm{e}} 1$-h ET-1 treatment $(1,5$, 10, 20 or $30 \mathrm{nM})$; ${ }^{\mathrm{f}} 2$-h ET-1 treatment $(1,5,10,20$ or $30 \mathrm{nM})$. OA, osteoarthritis; ET-1, endothelin-1; BQ123, endothelin receptor type A antagonist; BQ788, endothelin receptor type B antagonist; BKM120, phosphatidylinositol 3-kinase inhibitor.

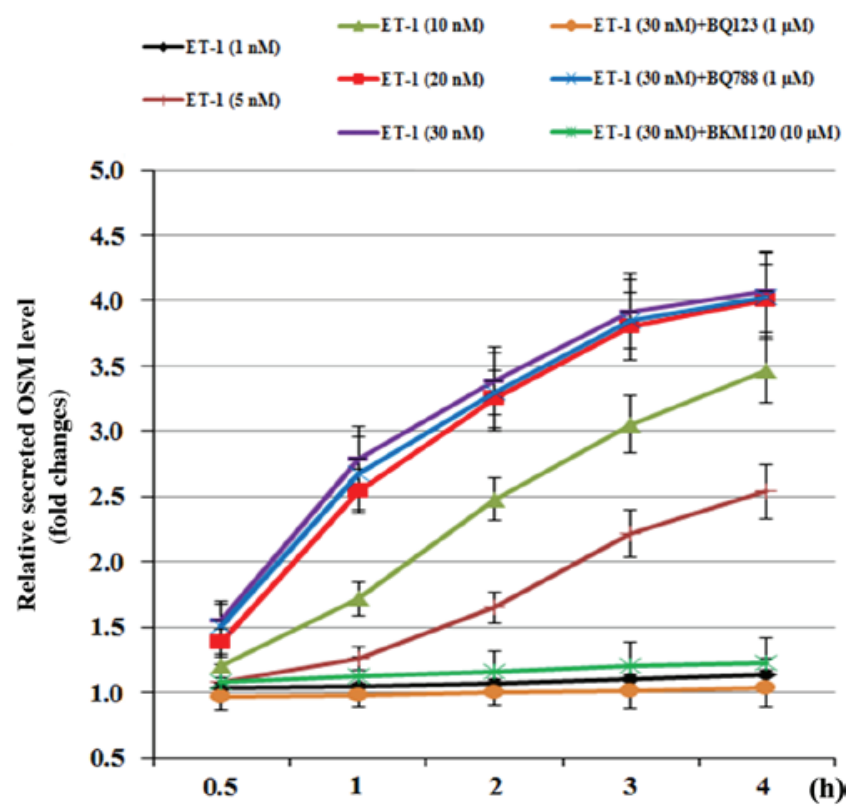

Figure 1. Secreted OSM levels of human OA osteoblasts treated with ET-1 in the presence and absence of ET receptor antagonists (BQ123, BQ788 and BKM120). OSM levels are demonstrated as the fold change of that of untreated control cells (designated as 1). ET-1, endothelin-1; OSM, oncostatin M; OA, osteoarthritis; BQ123, endothelin receptor type A antagonist; BQ788, endothelin receptor type B antagonist; BKM120, phosphatidylinositol 3-kinase inhibitor.

$24 \mathrm{~h}$ prior to western blotting and EMSAs, according to the manufacturer's protocol.

Statistical analysis. Statistical analyses were performed with SPSS software for Windows, version 19.0 (IBM SPSS, Armonk, NY, USA). Data are expressed as the mean \pm standard deviation. Comparisons of means among multiple groups were performed with one-way analysis of variance, followed by post-hoc pairwise comparisons using Tukey's test. Tests were two-tailed and $\mathrm{P}<0.05$ was considered to indicate a statistically significant difference.
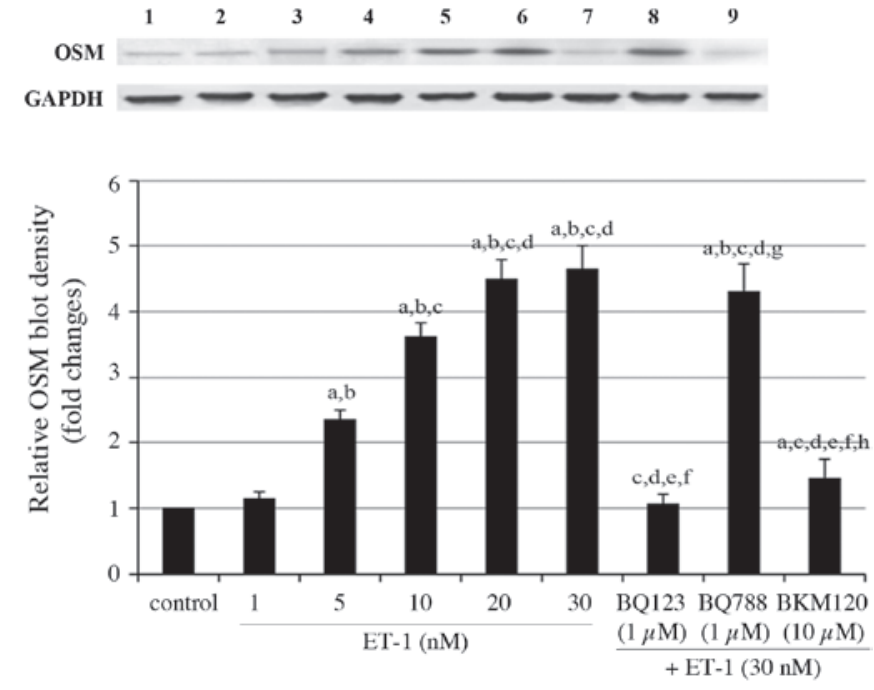

Figure 2. OSM protein levels in human OA osteoblasts treated with ET-1 in the presence and absence of ET receptor antagonists. Data were normalized to GAPDH and are expressed as fold changes to that of untreated control cells (designated as 1). Lanes were loaded in the order demonstrated on the graph. ${ }^{\text {a }} \mathrm{P}<0.05$ vs. the untreated control group; ${ }^{b} \mathrm{P}<0.05$ vs. the ET- 1 $(1 \mathrm{nM})$ group; ${ }^{\mathrm{C}} \mathrm{P}<0.05$ vs. the ET-1 $(5 \mathrm{nM})$ group; ${ }^{\mathrm{d}} \mathrm{P}<0.05$ vs. the ET- 1 $(10 \mathrm{nM})$ group; ${ }^{e} \mathrm{P}<0.05$ vs. the ET-1 $(20 \mathrm{nM})$ group; ${ }^{\mathrm{f}} \mathrm{P}<0.05$ vs. the ET-1 $(30 \mathrm{nM})$ group; ${ }^{\mathrm{g}} \mathrm{P}<0.05$ vs. the ET-1 $(30 \mathrm{nM})+\mathrm{BQ} 123(1 \mu \mathrm{M})$ group; and ${ }^{\mathrm{h}} \mathrm{P}<0.05$ vs. the ET-1 $(30 \mathrm{nM})+\mathrm{BQ788}(1 \mu \mathrm{M})$ group. OSM, oncostatin M; ET-1, endothelin-1; OA, osteoarthritis; BQ123, endothelin receptor type A antagonist; BQ788, endothelin receptor type B antagonist; BKM120, phosphatidylinositol 3-kinase inhibitor.

\section{Results}

ET-1 elevates the secreted OSM level of OA osteoblasts by inducing OSM mRNA expression levels. To investigate the potential effect of ET-1 on OSM expression in OA osteoblasts, primary human OA osteoblasts were treated with ET-1 $(1,5,10,20$ or $30 \mathrm{nM})$ for $0.5,1,2,3$ and $4 \mathrm{~h}$. The OSM mRNA and secreted protein levels were determined 26-29.5 h subsequent to treatment, making the total experimental time 

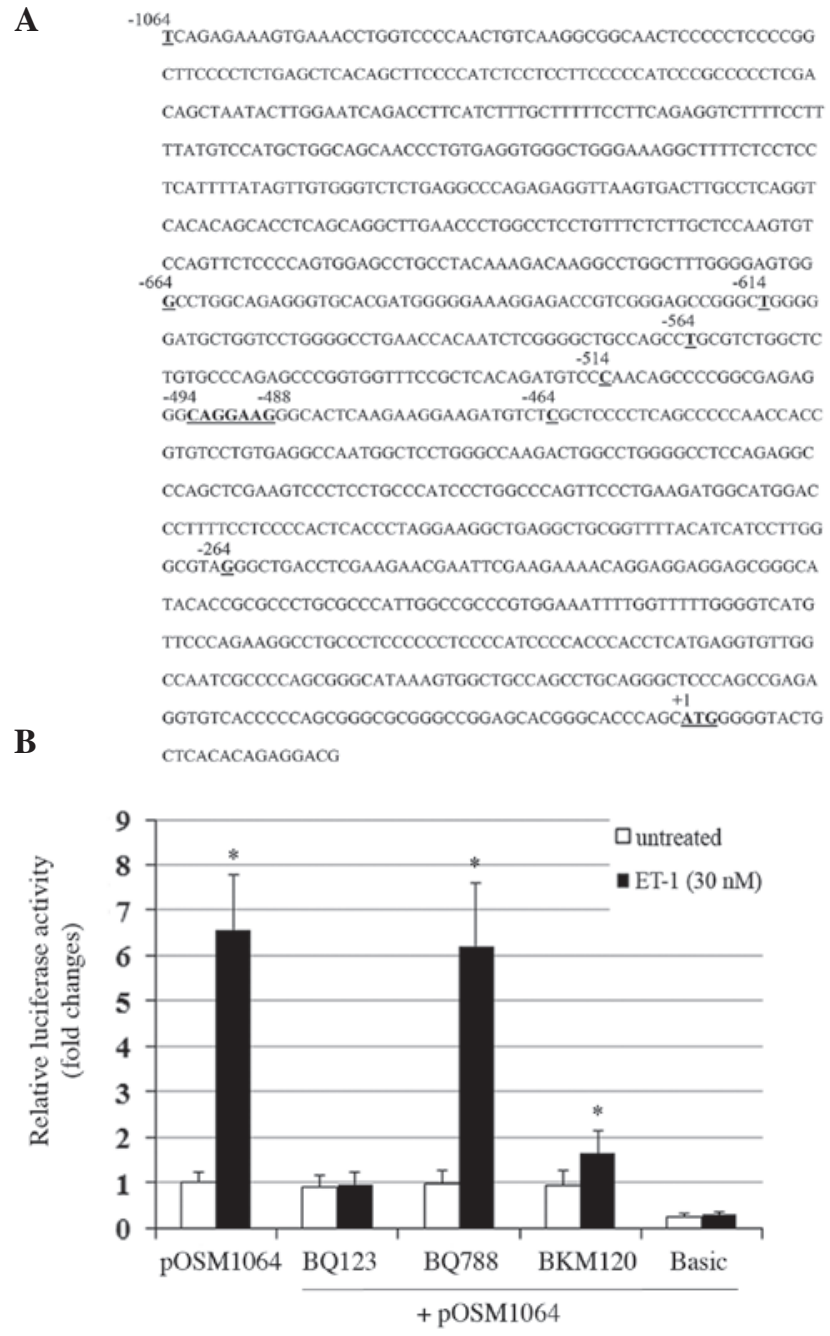

Figure 3. OSM gene promoter activities in human OA osteoblasts treated with ET-1 in the presence and absence of ET receptor antagonists (BQ123, BQ788 and BKM120). (A) A 1,064-bp human OSM gene promoter sequence. The ATG translation start codon is marked as +1 , and an Ets-1 binding site at -494/-488 and the start sites of 5'-end deletion constructs of the OSM promoter/luciferase reporter (pOSM1064) are underlined in bold. (B) pOSM1064 was used to assess the basic luciferase activity control. The constructs were transfected into human OA osteoblasts, and treated with $30 \mathrm{nM}$ ET-1 and BQ123 $(1 \mu \mathrm{M})$, BQ788 $(1 \mu \mathrm{M})$ and BKM120 $(10 \mu \mathrm{M})$ for $4 \mathrm{~h}$. Luciferase activity was measured $26 \mathrm{~h}$ later and expressed as the fold change of that of pOSM1064 in the untreated cells (designated as 1 ). ${ }^{*} \mathrm{P}<0.05$ vs. the untreated group. ET-1, endothelin-1; OSM, oncostatin M; OA, osteoarthritis; BQ123, endothelin receptor type A antagonist; BQ788, endothelin receptor type B antagonist; BKM120, phosphatidylinositol 3-kinase inhibitor; basic, luciferase reporter without the inserted promoter sequence.

$30 \mathrm{~h}$. As demonstrated in Table I, 1-20 nM ET-1 treatment significantly increased the OSM mRNA levels within $3 \mathrm{~h}$ in a dose- and time-dependent manner, and this effect was suppressed by the ETAR antagonist, BQ123 and PI3K inhibitor, BKM120, however not the ETBR antagonist, BQ788. ELISA assays demonstrated that the data trend for the secreted OSM level of the cell culture supernatants was similar to that of the OSM mRNA expression level (Fig. 1).

As demonstrated in Fig. 2, western blot analysis verified that intracellular protein levels of OSM in OA osteoblasts treated with ET-1 $(1,5,10,20$ or $30 \mathrm{nM})$ for $4 \mathrm{~h}$ were consistent with the corresponding OSM mRNA levels
A

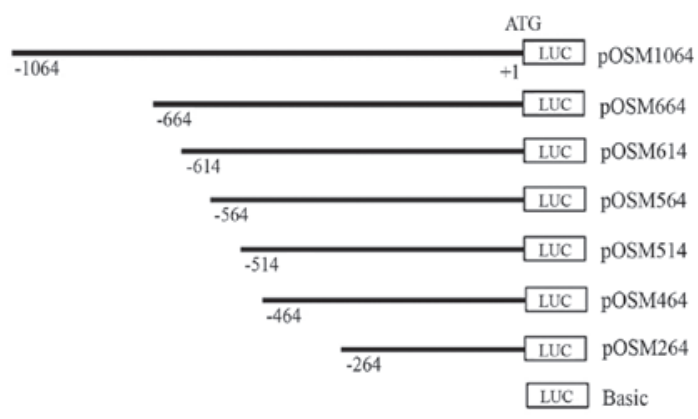

B

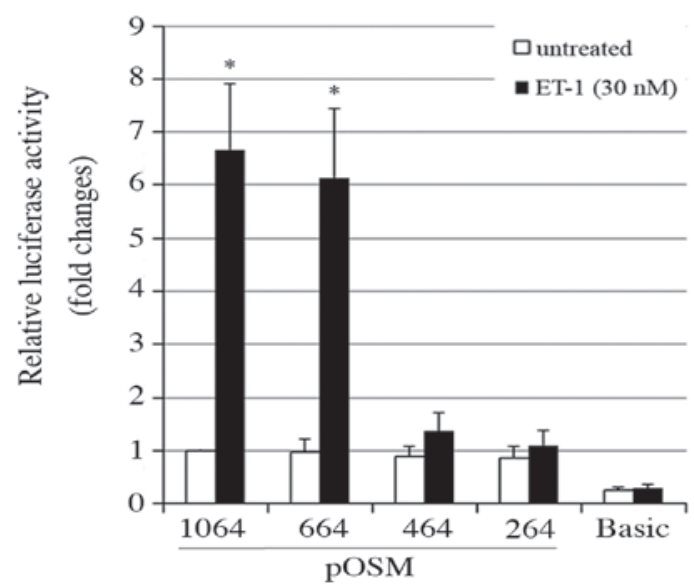

C

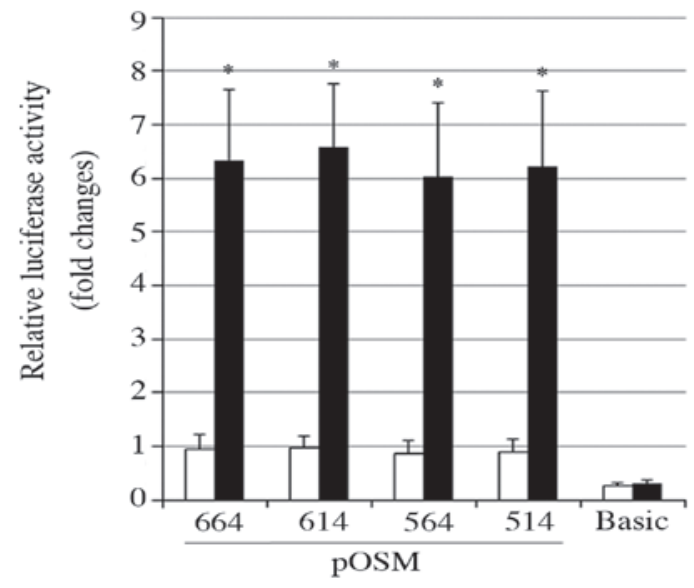

Figure 4. Deletional analysis of the human OSM gene promoter activity in ET-1-treated OA osteoblasts. The cells were untreated or treated with $30 \mathrm{nM}$ ET-1 for $4 \mathrm{~h}$. (A) Schematic representation of the human OSM gene promoter regions that were included in a series of 5'-end deletion constructs of the human OSM promoter/luciferase reporter. Each construct was designated by the relative position of the 5'-end nucleotide of the inserted OSM promoter region, with the ATG translation start codon marked as +1 . (B) 5'-end deletion constructs pOSM1064, -664, -464, -264 and (C) pOSM664, -614, -564, -514 and Basic were transfected into human OA osteoblasts. The luciferase activity assays were performed $26 \mathrm{~h}$ later and results are expressed as the fold change of that of pOSM1064 in the untreated cells (designated as 1). ${ }^{*} \mathrm{P}<0.05$ vs. the untreated groups. ET-1, endothelin-1; OSM, oncostatin M; OA, osteoarthritis; basic, luciferase reporter without the inserted promoter sequence.

(Table I) and secreted protein levels (Fig. 1). The results suggested that ET-1 may elevate the secreted OSM levels of OA osteoblasts by inducing OSM mRNA expression in an ETAR- and PI3K-dependent manner. 


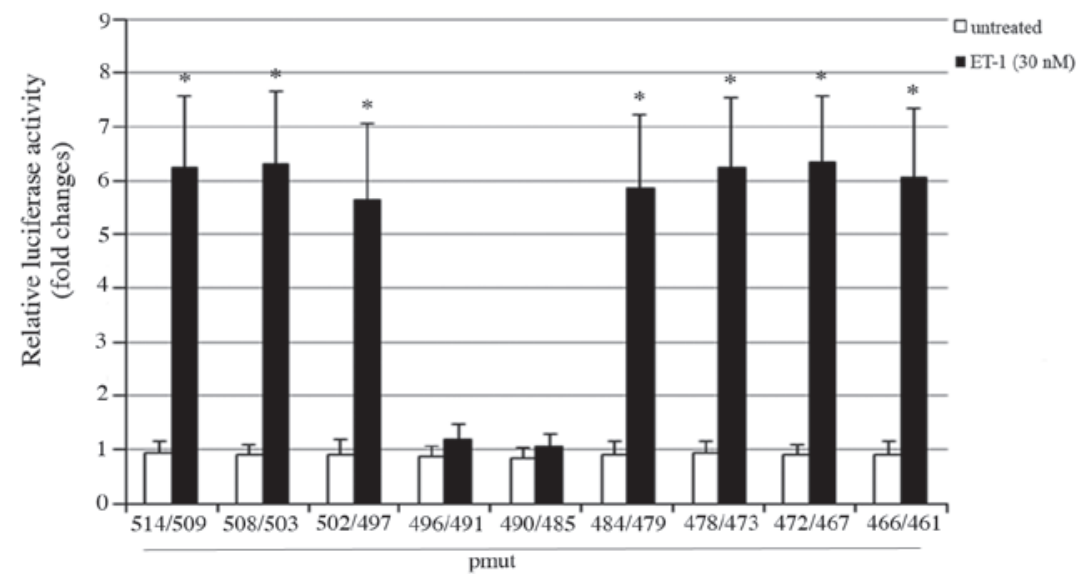

Figure 5. Mutational analysis of the human OSM gene promoter activity in ET-1-treated OA osteoblasts ( $30 \mathrm{nM} \mathrm{ET-1;} 4$ h). The human OSM gene promoter region from -514 to -464 in pOSM1064 was mutated with the EcoRI linker, GAATTC and the linker scan mutant constructs were transfected into human OA osteoblasts. The luciferase activity assay was performed $26 \mathrm{~h}$ later and results are expressed as the fold change of that of pOSM1064 in the untreated cells (designated as 1). $\mathrm{P}<0.05$ vs. the untreated groups. ET-1, endothelin-1; OSM, oncostatin M; OA, osteoarthritis.

ET-1 trans-activates the OSM gene promoter by increasing the specificity of Ets-1 binding to the promoter in OA osteoblasts. To examine whether ET-1 induced OSM mRNA expression by trans-activating the OSM gene promoter, the human OSM gene promoter activities were investigated using a commercial human OSM promoter/luciferase reporter, which contained $1,064 \mathrm{bp}$ of 5'-untranslated region directly upstream of the OSM gene translation start codon (Fig. 3A). This gene promoter sequence was inserted in frame with the luciferase cDNA and termed pOSM1064. The luciferase reporter without the inserted promoter sequence served as a basic luciferase activity control. Luciferase assays demonstrated that compared with the basic control, the OSM promoter was constitutively active in OA osteoblasts. ET-1 treatment markedly increased the OSM promoter activity, which was suppressed by BQ123 and BKM120, but not BQ788 (Fig. 3B). The results indicate that ET-1 trans-activates the OSM promoter in OA osteoblasts in an ETAR- and PI3K-dependent manner.

To identify the potential ET1-responsive cis-DNA element in the OSM promoter, a deletional analysis of the OSM promoter activity was conducted. As demonstrated in Fig. 4A, a series of 5'-end deletion constructs of the human OSM promoter/luciferase reporter were established. As demonstrated in Fig. 4B, the luciferase assays suggested that the OSM promoter region between -664 and -464 bp contained potential ET1-responsive cis-DNA elements. Further luciferase assays suggested that the cis-DNA element was between -514 and $-464 \mathrm{bp}$ in the OSM promoter (Fig. 4C). In addition, a mutational analysis of the OSM promoter activity was performed. A series of mutant OSM promoter reporters were generated by introducing mutations into the OSM promoter region between -514 and -464 bp in pOSM1064 with an EcoRI linker (GAATTC) at 6-bp intervals. As demonstrated in Fig. 5, mutations in the promoter region between -496 and -485 bp suppressed the trans-activating effect of ET-1 on the OSM promoter. Analysis of this region with the online software, PROMO $(11,12)$ revealed a consensus Ets-1 binding site between -494 and -488 bp (Fig. 6A).

EMSAs were conducted to examine whether Ets-1 specifically binds to the $-494 /-488$ putative Ets-1 binding site.
A
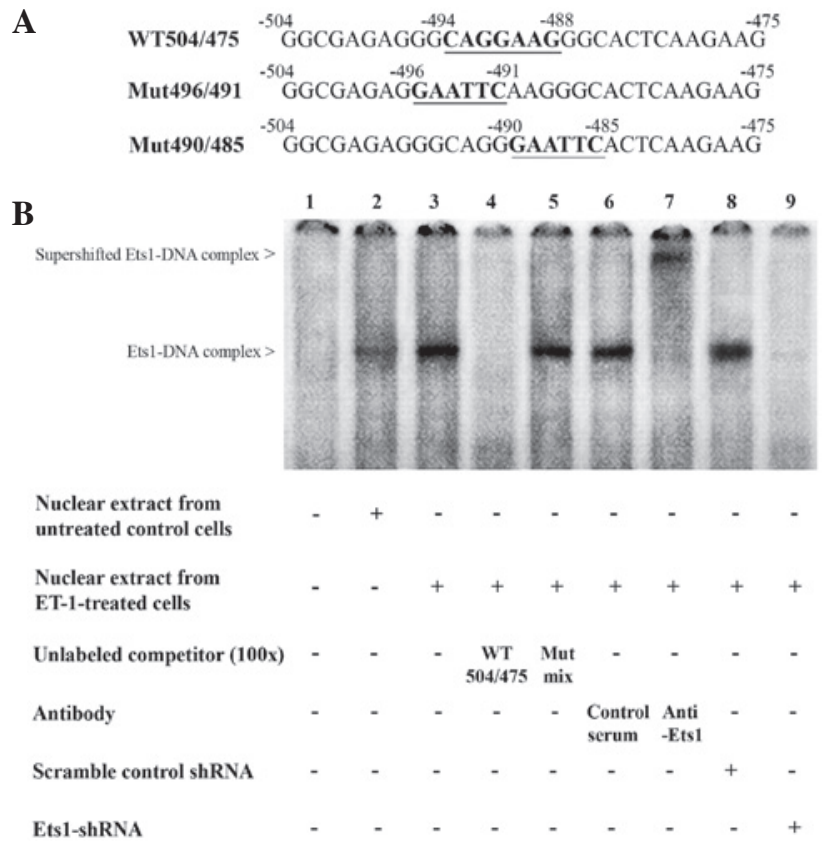

Figure 6. Specific protein-binding activity at the putative Ets-1 binding site in the human OSM gene promoter. (A) Oligonucleotide WT504/475 contained the human OSM gene promoter sequence from -504 to -475 encompassing the $-494 /-488$ putative Ets-1 binding site (underlined in bold). Oligonucleotides Mut496/491 and Mut490/485 contained the same sequence as WT504/475 except for the EcoRI linker (GAATTC) mutations at -496/-491 and -490/-485 (underlined in bold), respectively. (B) Electrophoretic mobility shift assays were performed using WT504/475 as the radiolabeled probe in the presence of equal quantities of nuclear extract from human OA osteoblasts. Lane 1, radiolabeled probe only; lanes 2-3, control reaction; lane 4, 100-fold molar excess of unlabeled oligonucleotide WT504/475 as a competitor; lane 5, mixture of 100-fold molar excess of each of unlabeled oligonucleotides, Mut496/491 and Mut490/485 as competitors; lane 6, control serum; lane 7, anti-Ets-1 antibody; lane 8, cells transduced with lentiviral scramble control shRNA; lane 9, cells transduced with lentiviral Ets-1 shRNA. Major protein-DNA and supershifted complexes are indicated by arrows. ET-1, endothelin-1; shRNA, small hairpin RNA; OSM, oncostatin M; OA, osteoarthritis; WT, wild-type; Mut, mutant.

Oligonucleotide WT504/475 corresponding to the human OSM promoter sequence between -504 and -475 bp was radiolabeled and used as the probe to incubate with OA osteoblast cell 
A
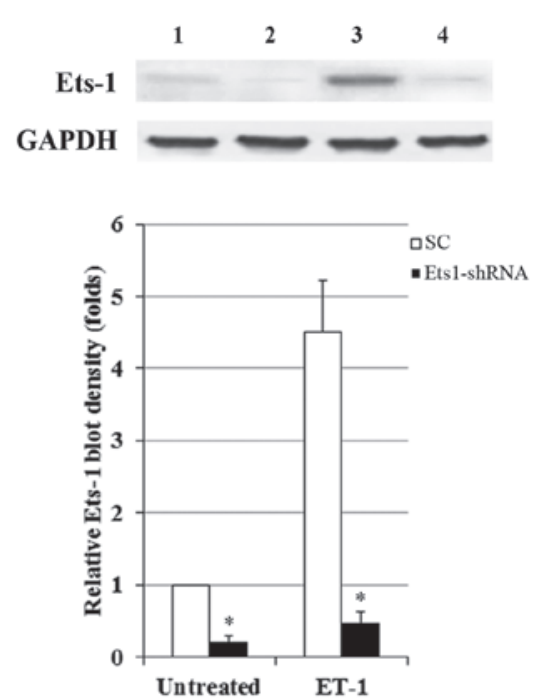

B
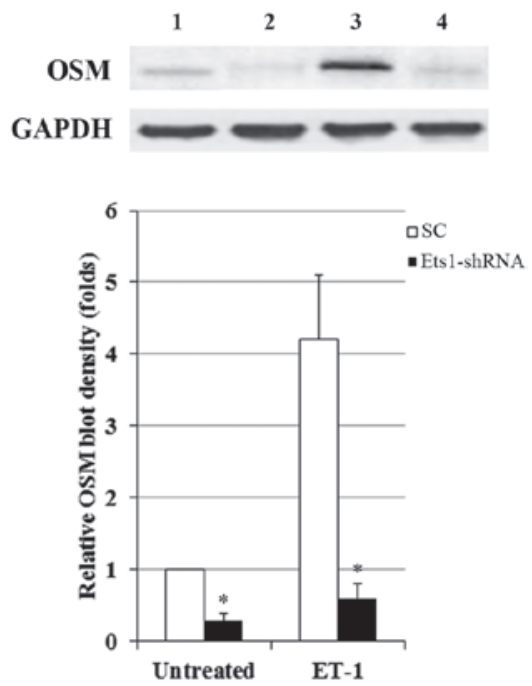

Figure 7. Effect of Ets-1 knockdown on the expression of OSM in ET-1-treated OA osteoblasts. Western blot analysis of (A) Ets-1 and (B) OSM protein expression levels following treatment. Ets-1 and OSM expression levels were normalized to that of GAPDH and expressed as the fold change of untreated SC (designated as 1). Cells were transduced with: Lane 1, SC; Lane 2, Ets1-shRNA; Lane 3, SC and treatment with ET-1 (30 nM) for $4 \mathrm{~h}$; Lane 4, Ets1-shRNA and treatment with ET-1 (30 nM) for $4 \mathrm{~h} .{ }^{*} \mathrm{P}<0.05$ vs. the untreated groups. ET-1, endothelin-1; OSM, oncostatin M; OA, osteoarthritis; SC, scramble control shRNA; shRNA, small hairpin RNA.

nuclear extract in the EMSAs (Fig. 6A). Unlabeled WT504/475, and Mut496/491 and Mut490/485, two oligonucleotides with the same sequence as WT504/475 except for the EcoRI linker (GAATTC) mutations at -496/-491 and -490/-485, respectively, served as competitors to the probe (Fig. 6A). As demonstrated in Fig. 6B, nuclear extract from ET-1-treated cells indicated a markedly stronger binding activity with the probe compared with that of the untreated control cells. A 100-fold molar excess of unlabeled WT504/475 (but not the mixture of 100-fold molar excess of each of Mut496/491 and Mut490/485) suppressed the binding activity (Fig. 6B), suggesting specific protein binding at the -494/-488 putative Ets-1 binding site. Furthermore, the control serum exerted no effect, whereas the anti-Ets1 antibody supershifted the major protein-DNA complex to a higher position (Fig. 6B). The results indicate that ET-1 may markedly

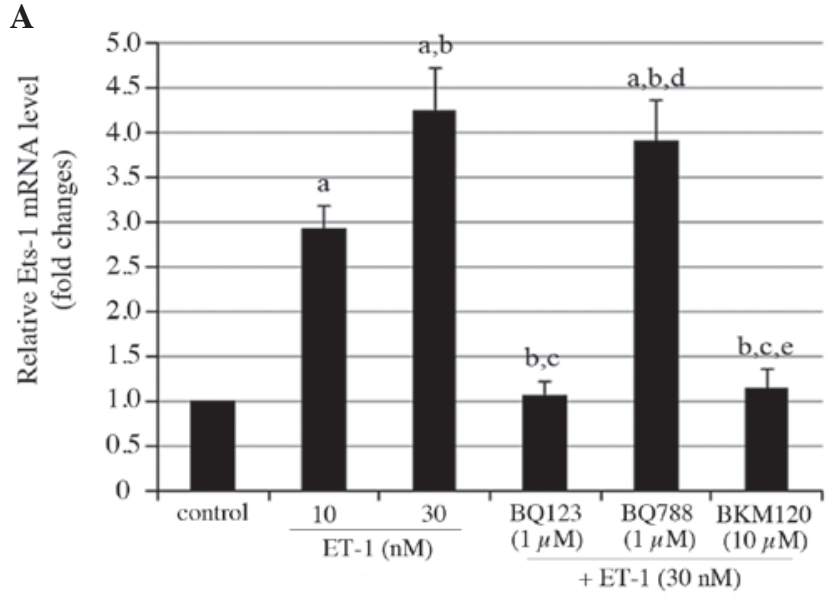

B
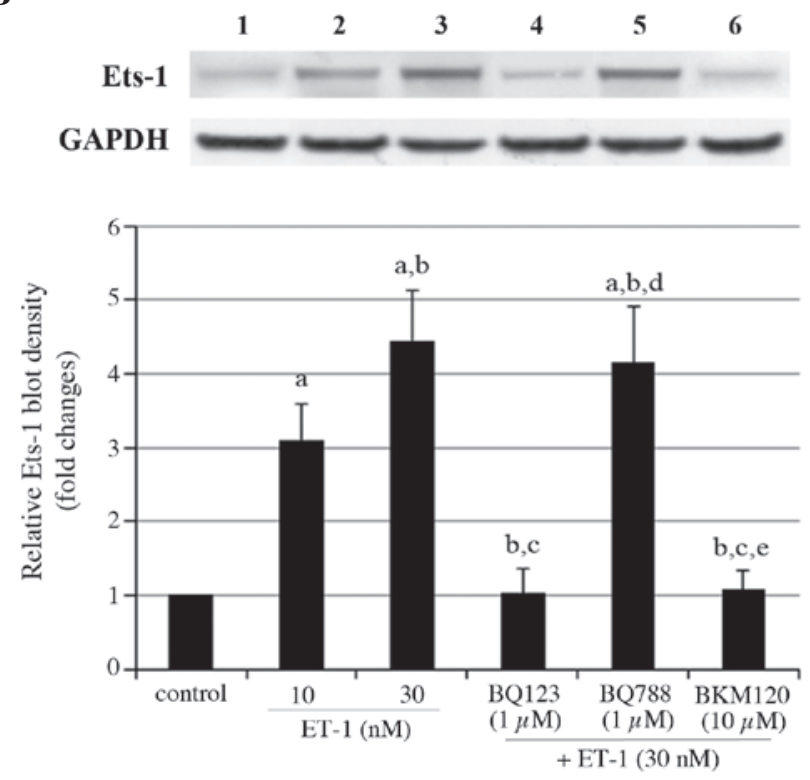

Figure 8. Ets-1 mRNA and protein expression levels in human OA osteoblasts treated with ET-1 in the presence and absence of ET receptor antagonists (BQ123, BQ788 and BKM120). (A) Ets-1 mRNA expression levels following treatment.(B) Western blot analysis of Ets-1 protein expression levels following treatment. Ets-1 expression levels were normalized to that of GAPDH and are expressed as the fold change of that of untreated control cells (designated as 1). Lanes were loaded in the same order as indicated in the graph. $\mathrm{P}<0.05$ vs. the ${ }^{\mathrm{a}}$ control; ${ }^{\mathrm{b}} \mathrm{ET}-1$ (10 nM); ${ }^{\mathrm{C} T}$-1 (30 nM); ${ }^{\mathrm{d}} \mathrm{ET}-1$ + BQ123; and ${ }^{\mathrm{e}} \mathrm{ET}-1$ + BQ788 groups. OSM, oncostatin M; ET-1, endothelin-1; OA, osteoarthritis; BQ123, endothelin receptor type A antagonist; BQ788, endothelin receptor type B antagonist; BKM120, phosphatidylinositol 3-kinase inhibitor.

increase specific binding of Ets-1 to the -494/-488 region in the OSM promoter in OA osteoblasts. In addition, the Ets1-DNA complex was abolished by Ets-1 shRNA, and not the scramble control shRNA, further confirming the specificity of the Ets1-DNA complex.

Ets-1 mediates the inducing effect of ET-1 on OSM expression in OA osteoblasts. To determine the functional role of Ets-1 in the ET1-induced expression of OSM, the activity of Ets-1 in OA osteoblasts was knocked down with lentiviral shRNA. As demonstrated in Fig. 7A, the protein levels of Ets-1 were markedly increased by ET-1, compared with the Ets-1-shRNA group. However, transduction of Ets-1-shRNA significantly decreased the Ets-1 protein level by $\sim 80 \%$, in the presence and 
absence of ET-1 $(\mathrm{P}<0.05)$. As demonstrated in Fig. 7B, Ets-1 knockdown suppressed the ET1-induced expression of OSM. The results indicate that Ets-1 is an important mediator of the inducing effect of ET-1 on OSM expression in OA osteoblasts.

Effect of ET-1 on the protein expression of Ets-1. OA osteoblasts were treated with ET-1 (10 and $30 \mathrm{nM})$ for $4 \mathrm{~h}$ to assess the effect of ET-1 on the Ets-1 mRNA and protein expression levels. As demonstrated in Fig. 8, ET-1 treatment (10 and $30 \mathrm{nM}$ ) significantly increased the expression of Ets-1 at the mRNA and protein levels compared with the control group $(\mathrm{P}<0.05)$. This effect was significantly suppressed following co-treatment with BQ123 or BKM120, compared with the control and ET-1 only groups (Fig. 8; P<0.05). However, co-treatment with BQ788 significantly increased the expression of Ets-1 compared with the control and ET-1 only groups (Fig. 8; $\mathrm{P}<0.05$ ). These findings indicate that ET-1 may increase Ets-1 expression levels in OA osteoblasts in an ETAR- and PI3K-dependent manner.

\section{Discussion}

OSM is an established contributor to cartilage degeneration in OA (2-4) and is expressed in OA osteoblasts $(9,10)$. ET-1 is implicated in the degradation of OA articular cartilage $(6,7)$, and involved in osteoblast proliferation and bone development $(5,8)$. The present study demonstrated that ET-1 induced the expression of OSM in OA osteoblasts.

The ET family consists of ET-1, -2 , and -3 and the endothelin-converting enzymes (ECEs) that catalyze the generation of biologically active ETs (15). The roles of ET-2 and -3 , and ECE-1 in the expression of OSM and Ets- 1 in OA osteoblasts remain unclear. In addition, the effect of ET-1 on the expression of OSM in normal osteoblasts is yet to be determined.

ET-1 treatment (5-20 nM) for $\geq 1 \mathrm{~h}$ dose-dependently induced the expression of OSM in OA osteoblasts. In particular, 20 or $30 \mathrm{nM}$ ET-1 induced mRNA and secreted protein levels of OSM by 4-fold within $30 \mathrm{~h}$, indicating that ET-1 is an inducer of OSM in OA osteoblasts. As the important role of OSM in the pathogenesis and progression of OA is well known (2-4), the results of the present study revealed, to the best of our knowledge, the first evidence of the mechanistic role of ET-1 in the pathophysiology of OA. Furthermore, ETAR antagonist, BQ123 significantly suppressed the ET-1-induced effect on the expression of OSM in OA osteoblasts, thus ETAR antagonists may be beneficial for patients with OA. The effects of ETAR antagonists on $\mathrm{OA}$ require further investigation in vivo.

The present study demonstrated that specific binding of Ets-1 to a -494/-488 cis-DNA element in the OSM gene promoter was required for the ET1-induced expression of OSM in OA osteoblasts, and that ET-1 dose-dependently increased the expression level of Ets-1 in OA osteoblasts. The results suggested that ET-1 induced the expression of OSM in OA osteoblasts primarily via inducing Ets-1 expression and Ets-1-dependent trans-activation of the OSM gene promoter. Furthermore, ET-1 induced the expression of Ets-1 in an ETAR- and PI3K-dependent manner, which explains why ET-1 induced OSM expression in OA osteoblasts. Future studies are required to investigate how the administration of ET-1 results in the increased expression of Ets-1 in OA osteoblasts via the PI3K signaling pathway.

A previous study demonstrated that ET-1 induced the expression of Ets-1 in primary proximal tubule cells (16), which is consistent with the observations of the OA osteoblasts in the present study. However, in the current study, the inducing effect of ET-1 on the expression of Ets-1 was via an ETAR, compared with the previous study, which demonstrated that the effect of ET-1 was via an ETAR and, more markedly, an ETBR (16). This discrepancy may be due to the different cell models used in the studies.

ET-1 was demonstrated to induce the production of matrix metalloproteinase (MMP)-1 by articular chondrocytes and synoviocytes, by which it may trigger the enzymatic degradation of articular cartilage (5). Hence, ET-1 may be a target of novel therapeutic approaches for OA (5). Ets-1 is a transcription factor that regulates the gene expression of proteases, such as urokinase-type plasminogen activator and MMP-1 (17,18). The current study demonstrated that ET-1 induced the expression of Ets-1 in OA osteoblasts, thus whether ET-1 may induce the production of MMP-1 in OA osteoblasts via Ets-1 may be of interest for a future study. In addition to the above-mentioned finding, the ET1-induced expression of OSM in OA osteoblasts, and the MMP-1 in articular chondrocytes and synoviocytes, indicates that the role of ET-1 in contributing to the pathogenesis and progression of OA may be diverse.

In conclusion, the present study has demonstrated that ET-1 induces the expression of OSM in OA osteoblasts via trans-activation of the OSM gene promoter. ET-1 increased the expression/specific binding of Ets-1 to an Ets-1 binding site (-494 to -488) in the OSM promoter in an ETAR- and PI3K-dependent manner. It therefore provides a novel insight into the mechanistic role of ET-1 in the pathophysiology of OA.

\section{References}

1. Sin A, Tang W, Wen CY, Chung SK and Chiu KY: The emerging role of endothelin-1 in the pathogenesis of subchondral bone disturbance and osteoarthritis. Osteoarthritis Cartilage 23: 516-524, 2015.

2. Chen CY, Su CM, Huang YL, Tsai CH, Fuh LJ and Tang CH: CCN1 induces oncostatin $\mathrm{M}$ production in osteoblasts via integrin-dependent signal pathways. PLoS One 9: e106632, 2014.

3. Sims NA and Walsh NC: GP130 cytokines and bone remodelling in health and disease. BMB Rep 43: 513-523, 2010.

4. Walker EC, McGregor NE, Poulton IJ, Solano M, Pompolo S, Fernandes TJ, Constable MJ, Nicholson GC, Zhang JG, Nicola NA, et al: Oncostatin M promotes bone formation independently of resorption when signaling through leukemia inhibitory factor receptor in mice. J Clin Invest 120: 582-592, 2010.

5. Roy-Beaudry M, Martel-Pelletier J, Pelletier JP, M'Barek KN, Christgau S, Shipkolye F and Moldovan F: Endothelin 1 promotes osteoarthritic cartilage degradation via matrix metalloprotease 1 and matrix metalloprotease 13 induction. Arthritis Rheum 48: 2855-2864, 2003.

6. Tatrai A, Foster S, Lakatos P, Shankar G and Stern PH: Endothelin-1 actions on resorption, collagen and noncollagen protein synthesis, and phosphatidylinositol turnover in bone organ cultures. Endocrinology 131: 603-607, 1992.

7. Tatrai A, Lakatos P, Thompson S and Stern PH: Effects of endothelin-1 on signal transduction in UMR-106 osteoblastic cells. J Bone Miner Res 7: 1201-1209, 1992.

8. Manacu CA, Martel-Pelletier J, Roy-Beaudry M, Pelletier JP, Fernandes JC, Shipkolye FS, Mitrovic DR and Moldovan F: Endothelin-1 in osteoarthritic chondrocytes triggers nitric oxide production and upregulates collagenase production. Arthritis Res Ther 7: R324-332, 2005. 
9. Lisignoli G, Piacentini A, Toneguzzi S, Grassi F, Cocchini B, Ferruzzi A, Gualtieri G and Facchini A: Osteoblasts and stromal cells isolated from femora in rheumatoid arthritis (RA) and osteoarthritis (OA) patients express IL-11, leukaemia inhibitory factor and oncostatin M. Clin Exp Immunol 119: 346-353, 2000.

10. Lisignoli G, Toneguzzi S, Pozzi C, Piacentini A, Riccio M, Ferruzzi A, Gualtieri G and Facchini A: Proinflammatory cytokines and chemokine production and expression by human osteoblasts isolated from patients with rheumatoid arthritis and osteoarthritis. J Rheumatol 26: 791-799, 1999.

11. Messeguer X, Escudero R, Farré D, Núñez O, Martínez J and Albà MM: PROMO: Detection of known transcription regulatory elements using species-tailored searches. Bioinformatics 18 333-334, 2002.

12. Farré D, Roset R, Huerta M, Adsuara JE, Roselló L, Albà MM and Messeguer X: Identification of patterns in biological sequences at the ALGGEN server: PROMO and MALGEN. Nucleic Acids Res 31: 3651-3653, 2003.

13. Livak KJ and Schmittgen TD: Analysis of relative gene expression data using real-time quantitative PCR and the $2^{-\Delta \Delta C}$ method. Methods 25: 402-408, 2001.
14. Johnson DR, Levanat S and Bale AE: Direct molecular analysis of archival tumor tissue for loss of heterozygosity. Biotechniques 19: 190-192, 1995.

15. Nelson JB, Hedican SP, George DJ, Reddi AH, Piantadosi S, Eisenberger MA and Simons JW: Identification of endothelin-1 in the pathophysiology of metastatic adenocarcinoma of the prostate. Nat Med 1: 944-949, 1995.

16. Von Brandenstein M, Schlosser M, Richter C, Depping R and Fries JW: ETS-dependent p16INK4a and p21waf1/cip1 gene expression upon endothelin-1 stimulation in malignant versus and non-malignant proximal tubule cells. Life Sci 91: 562-571, 2012.

17. Iwasaka C, Tanaka K, Abe M and Sato Y: Ets-1 regulates angiogenesis by inducing the expression of urokinase-type plasminogen activator and matrix metalloproteinase- 1 and the migration of vascular endothelial cells. J Cell Physiol 169: $522-531,1996$.

18. Oda N, Abe M and Sato Y: ETS-1 converts endothelial cells to the angiogenic phenotype by inducing the expression of matrix metalloproteinases and integrin beta3. J Cell Physiol 178: 121-132, 1999. 